\title{
Proposal of a locally generated audible sound using ultrasonic waves
}

\author{
Yuu Kouno, ${ }^{\mathrm{a}, *}$, Masaharu Moritsugu ${ }^{\mathrm{a}}$, Seiichi Serikawa ${ }^{\mathrm{a}}$ \\ ${ }^{a}$ Kyushu Institute of Technology, 1-1 Sensui-cho, Tobata-ku, Kitakyushu-shi, Fukuoka, 804-8550, Japan \\ *Corresponding Author: kouno@boss.ecs.kyutech.ac.jp
}

\begin{abstract}
Currently, in museums and galleries, parametric loudspeakers are useful that can be heard in a local place as description guide. Parametric loudspeakers have high directivity, so multiple announcements are possible that can coexist with limited space. However, conventional parametric loudspeakers have the problem that the sound maybe be heard at unexpected places because of the reflection of ultrasonic wave by walls and people. Therefore, the location and direction of parametric loudspeakers must be limited. In this paper, we propose a local audible sound generation system using ultrasound. This system generates an aimed audible tone at a local space using the intersection of ultrasound. Thus, it can prevent the generation of audible sound by reflection at unexpected places and the sound is audible at expected local places.
\end{abstract}

Keywords: ultrasound, locally audible sound, parametric loudspeaker, intersection of ultrasound.

\section{Introduction}

Parametric loudspeakers have high directivity, so multiple announcements are possible that can coexist with limited space. Because of this properties, it is expected to be used in museums and galleries as multiple and individual announcements that can be heard at local places as description guide. However, conventional parametric loudspeakers have the problem that the sound maybe be heard at unexpected places because of the reflection of ultrasonic wave by walls and people. Therefore, the location and direction of parametric loudspeakers must be limited.

In this paper, we propose a local audible sound generation system using ultrasound. This system generates an aimed audible tone at a local space using the intersection of ultrasound. Thus, it can prevent the generation of audible sound by reflection at unexpected places and the sound is audible at expected local places.

\section{Principle}

\subsection{Constitution}

Figure 1 shows the overall structure of this system. Locally audible sound generating system uses two ultrasonic loudspeakers. The input of each ultrasonic loudspeaker uses the DSB signal and the carrier signal alternately, that is, if the input of one ultrasonic loudspeaker uses the DSB signal, the other one uses the carrier signal. In addition, the period to exchange the inputs of the two ultrasonic loudspeakers is selected near formant frequency in order to disturb the sound at unexpected paces. By using this constitution, the audible sound waves of the intersection of the two appear at the expected place.

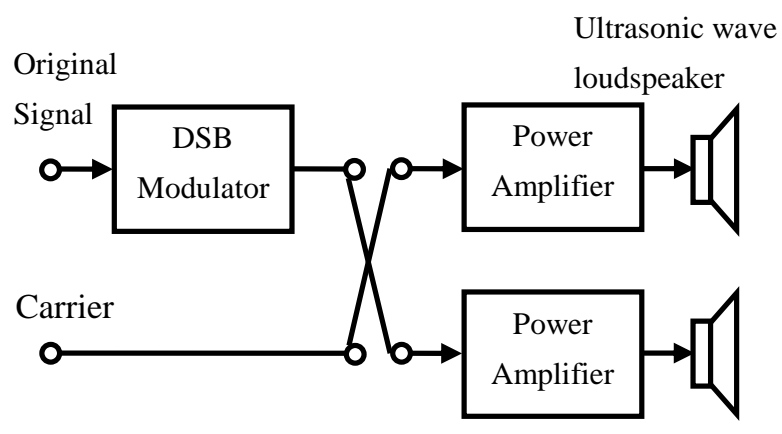

Fig. 1. System configuration

\subsection{Locally generated audible sound}

In order to generate an audible sound locally, the character of an ultrasonic wave and intersection of two waves are used. The character of an ultrasonic wave is 
giving directivity to sound. As general characterization of a wave including the sound of the ultrasonic wave, directivity becomes settled in the ratio of the size of the vibrating surface which is a wave source to the wavelength of the wave to leave. That is, although sound goes away almost uniformly that the diameter of a vibrating surface is a size below a wavelength grade for all directions, sound has the character concentrated and released in the direction of the front, so that a vibrating surface is large compared with a wavelength. Directivity is given to the sound emitted from an ultrasonic loudspeaker by this. The ultrasonic loudspeaker to be used has a circular opening of the diameter $13[\mathrm{~mm}]$ and the frequency is $40[\mathrm{kHz}]$. Equation (1) shows the relationship between the wavelength and frequency.

$$
\lambda=\frac{v}{f}
$$

where, $v$ is the propagation speed of sound in the air, $f$ is the frequency, $\lambda$ represents the wavelength.

The intersection of two waves uses two wave-like different waves emitted from two ultrasonic loudspeakers with the same frequency, and the sound of the original signal appears only at the place with which they overlap. The envelope curve portion of the ultrasonic wave which overlaps serves as the same waveform as the original signal. And since the frequency of $40[\mathrm{kHz}]$ is outside human auditory sensation area, an envelope curve portion gets over with ear, and it can be heard.

Let $\omega_{1}$ and $\omega_{2}$ be the carrier wave frequency and that of the signal, respectively. Then the signal wave $X$ modulated by carrier wave and the carrier wave $Y$ can be expressed as Eqs. (2) and (3), respectively.

$$
\begin{aligned}
X & =\sin \omega_{1} t \cdot \cos \omega_{2} t \\
& =\frac{1}{2}\left\{\sin \left(\omega_{1}+\omega_{2}\right) t+\sin \left(\omega_{1}-\omega_{2}\right) t\right\} \\
Y & =\sin \omega_{1} t
\end{aligned}
$$

The superposition of these two waves becomes

$$
X+Y=\sin \omega_{1} t\left(\cos \omega_{2} t+1\right)
$$

\subsection{Formant frequency}

The wave of Eq. (4) occurs where wave Eq. (3) is overlapped with the Eq. (2) exactly. The original signal is detected in human ear. However, the sound detected only from the modulated signal wave of Eq. (2) is also heard close to the original signal by human ear. So, it can be considered if the formant frequencies of Eq. (2), the original signal may be not heard only from the wave of Eq. (2). Formant frequency is the frequency important to identify the vowel. In other words, if the formant frequencies are removed from audio signal, it will not be able to recognize the vowel and it will not be able to understand the contents of speech.

As a method for removing this formant frequency, it is performed at intervals of formant frequency to exchange the input waves of two loudspeakers with the wave of Eqs. (3) and (2).

\section{Experiment}

\subsection{Experimental method}

\section{(a) Preliminary experiment}

Before the experiment using the ultrasonic speaker, we performed preliminary experiments using a normal speaker in order to obtain the correlation of the system. Original signal emitted from the speaker is received by a condenser microphone. The correlation between the received signal and the original signal is measured.

The construction of preliminary experiment is shown in Fig. 2. The signal processing steps are shown in Fig. 3. The

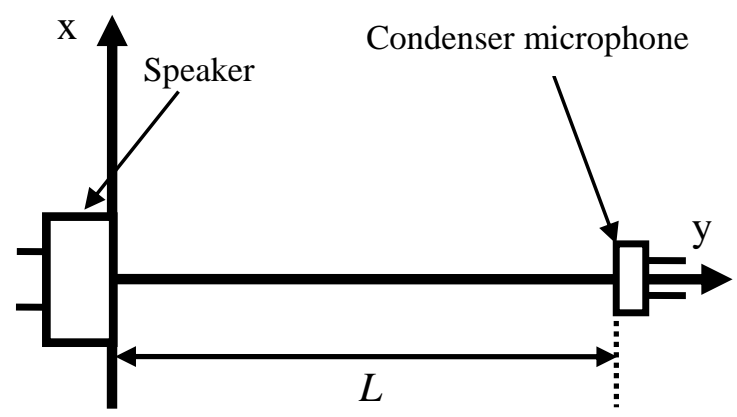

Fig. 2. Configuration of Preliminary experiment

Input Signal

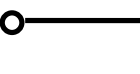

The received

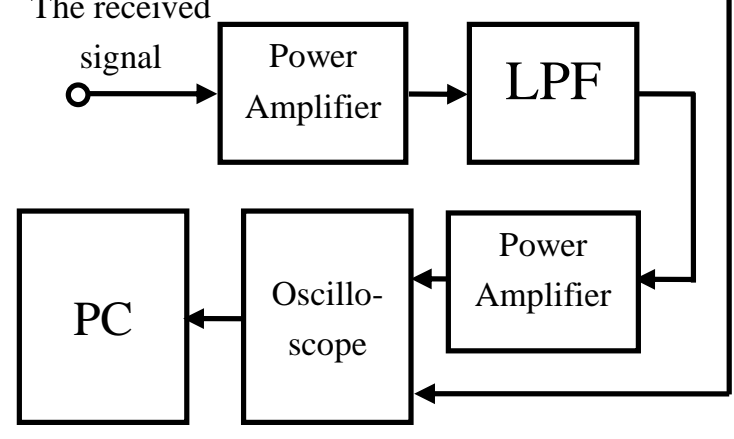

Fig. 3. Signal processing 
received the sound signal by the condenser microphone is processed through an amplifier, the LPF, and finally, the signal is input into a PC. Incorporated in a similar manner the waveform of the original signal is input at the same time. We compared by taking the correlation between this two waveforms captured on PC. Here, the distance of the transmitter and receiver is $L \doteqdot 4.33[\mathrm{~cm}]$ in the experiment.

(b) Experiment

The experimental measuring system is shown in Fig. 4. Around its radial axis of the loudspeaker portion of the two ultrasonic loudspeakers, measurements were carried out at various positions to compare received signal to the original one. The distance between the two ultrasonic speakers is $d$ $=5[\mathrm{~cm}]$, and the angles between the $y$-axis and the radial axes of the two ultrasonic speakers are $\theta=30\left[^{\circ}\right]$ as shown in Fig. 4. The measurement points were moved with the interval $1[\mathrm{~cm}]$ from the intersection point of the radial axis. And measurement points extents are $-5 \sim+5[\mathrm{~cm}]$ along $\mathrm{x}$-axis and -3 to $+5[\mathrm{~cm}]$ along $\mathrm{y}$-axis.

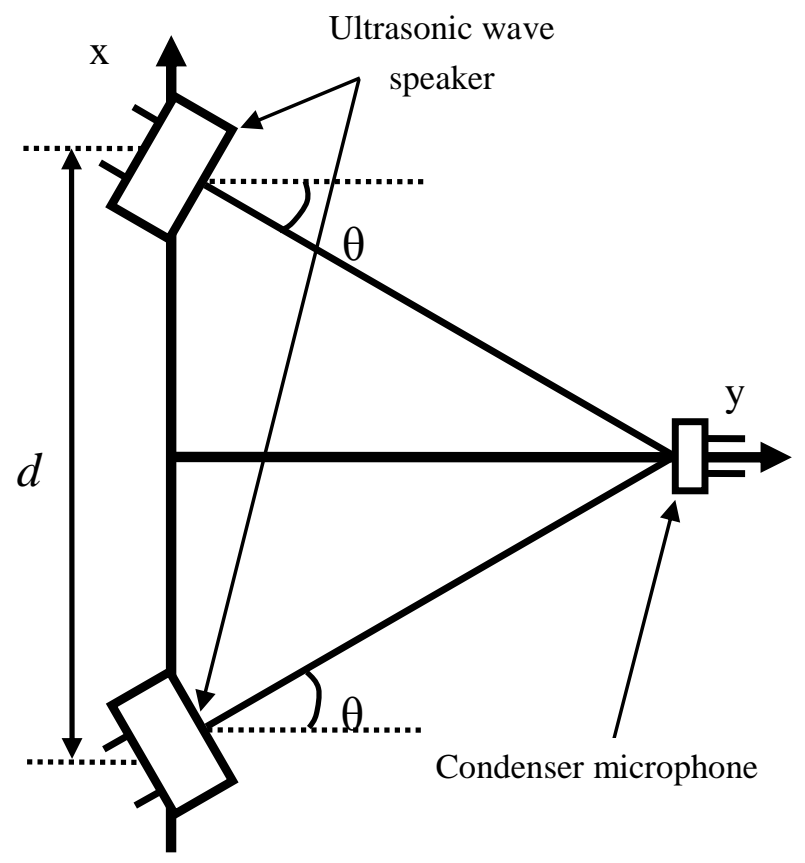

Fig. 4. Measuring system configuration of experiment

\subsection{Experimental Results}

In the preliminary experiment, the normalized correlation coefficient was measure as a value of 0.8972 . This means the measuring system is not perfect because of the noise caused in the system.

Experimental results using the measuring system shown in Fig. 4 are shown in table 1 and Fig. 4.
Table 1. Normalized correlation coefficients (a) $\mathrm{x}$-axis

\begin{tabular}{|c|c|}
\hline $\mathrm{x}[\mathrm{cm}]$ & correlation \\
\hline-5 & 0.393 \\
\hline-4 & 0.686 \\
\hline-3 & 0.845 \\
\hline-2 & 0.821 \\
\hline-1 & 0.905 \\
\hline 0 & 0.913 \\
\hline 1 & 0.891 \\
\hline 2 & 0.729 \\
\hline 3 & 0.684 \\
\hline 4 & 0.344 \\
\hline 5 & 0.46 \\
\hline
\end{tabular}

(b) y-axis

\begin{tabular}{|c|c|}
\hline $\mathrm{y}[\mathrm{cm}]$ & correlation \\
\hline-3 & 0.866 \\
\hline-2 & 0.851 \\
\hline-1 & 0.708 \\
\hline 0 & 0.913 \\
\hline 1 & 0.811 \\
\hline 2 & 0.705 \\
\hline 3 & 0.572 \\
\hline 4 & 0.863 \\
\hline 5 & 0.653 \\
\hline
\end{tabular}

For the $\mathrm{X}$-axis, the correlation is lower farther from the center. For the Y-axis, there is a periodic correlation. However, the correlation is lower as the distance from the center, too. Thus, the sound is close to the original signal only around the center point. From the above results, it maybe said a desired sound can be generated at local point.

\section{Conclusions}

In this study, we proposed a system to generate audible sound using ultrasonic waves. This system can generate a desired sound close to the original one only at the desired point. It overcame the problem that a near original sound is heard out of the desired position. As a further study, we will modify the measuring system to improve the accuracy and construct a system with multiple ultrasonic loudspeakers more than two. 


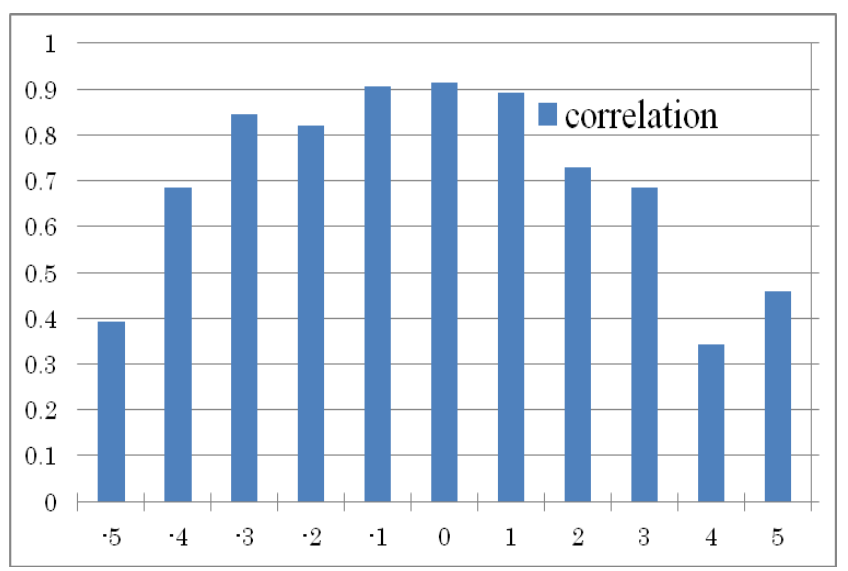

(a) $\mathrm{x}$-axis

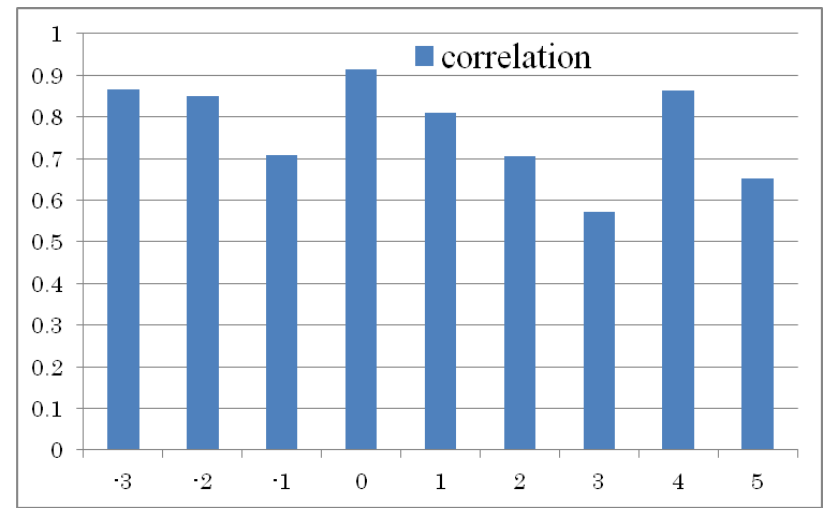

(b) y-axis

Fig. 4. Correlation

\section{References}

(1) Yoshiyuki Hara, Yoshu Maekawa, and Tomoo Kamakura : "Driving for Reducing Carrier Ultrasound of Parametric Loudspeaker", IEICE-US2010-1, IEICE-110, IEICE-US-10, pp. 1-4, 2010

(2) Akira Kikuchi, Yosyu Maekawa, Yoshiyuki Hara, and Tomoo Kamakura : "Out-of-phase excitation for parametric loudspeaker -Effect of arrangements of ultrasonic projectors on parametric fields-", IEICE-EA2010-1, IEICE-109, IEICE-EA-389, pp. 25-28, 2010

(3) Masayoshi Kondo, Daisuke Fukagawa, Hideyuki Nomura, and Tomoo Kamakura : "Phase inversion driving for parametric loudspeaker -Reduction of ultrasonic sound pressure and application for head model-", IEICE-US2009-1, IEICE-108, IEICE-US-410, pp. 33-36, 2009 